\title{
Effect of harness application and postural changes on cardiovascular parameters of individuals with spinal cord injury
}

\author{
AV Krassioukov and SJ Harkema \\ Spinal Cord (2006) 44, 814. doi:10.1038/sj.sc.3102017
}

Correction to: Spinal Cord (2006) 44, 780-786. doi:10.1038/sj.sc.3101952; published online June 27, 2006.

Since the above paper has been published online the authors have identified an error. In the Research participants paragraph of the Methods section, the number of individuals with SCI should read 11, and in Table 1 the individual A25 should not appear. The corrected details are shown below.

\section{Research participants}

The UCLA Institutional Review Board approved the experimental protocol for the present study and each subject signed an informed consent form before participating in the study. Nine able-bodied individuals and 12 individuals with SCI volunteered for this study (Table 1).

Table 1 Demographic and clinical data of research participants

\begin{tabular}{|c|c|c|c|c|c|}
\hline Subject ID & Sex & Age (years) & Injury level & $A S I A$ & Years post-injury \\
\hline A19 & $\mathrm{m}$ & 55 & $\mathrm{C} 5$ & A & 14.7 \\
\hline A 20 & $\mathrm{~m}$ & 24 & $\mathrm{C} 5$ & A & 0.7 \\
\hline A 21 & $\mathrm{~m}$ & 27 & $\mathrm{C} 5$ & A & 4.9 \\
\hline A 29 & $\mathrm{~m}$ & 22 & $\mathrm{C} 5$ & A & 1.0 \\
\hline A 26 & $\mathrm{~m}$ & 48 & C6 & A & 29.5 \\
\hline A 27 & $\mathrm{~m}$ & 25 & C6 & A & 7.8 \\
\hline A 24 & $\mathrm{~m}$ & 41 & $\mathrm{~T} 4$ & A & 2.1 \\
\hline A 23 & $\mathrm{f}$ & 26 & $\mathrm{~T} 4$ & A & 7.4 \\
\hline A 22 & $\mathrm{~m}$ & 28 & T6 & A & 0.8 \\
\hline A 28 & $\mathrm{~m}$ & 21 & T6 & A & 0.8 \\
\hline A 30 & $\mathrm{~m}$ & 33 & T6 & A & 0.5 \\
\hline
\end{tabular}

Subject characteristics: C, cervical; f, female; ID, identification; m, male; T, thoracic. 